\title{
FREQUÊNCIA DE INFECÇÃO DO SÍTIO CIRÚRGICO EM GASTRECTOMIA
}

\author{
Frequence of surgical site infection in gastrectomy \\ Frecuencia de infección de sitio quirurgico en gastrectomía
}

Vanessa de Brito Poveda

Cristina Maria Galvão

\begin{abstract}
Resumo
A presente investigação teve como objetivo identificar a freqüência de infecção do sítio cirúrgico (ISC), em paciente submetido a cirurgia eletiva de gastrectomia, potencialmente contaminada, na especialidade de Gastrocirurgia, no período compreendido de 1998 a 2002, em um hospital escola de Ribeirão Preto - SP. Realizou-se um estudo retrospectivo, por meio do levantamento de informações contidas nos prontuários médicos. Em 181 casos investigados, detectou-se a ocorrência de infecção do sítio cirúrgico em 17 pacientes (9,4\%), sendo 23,5\% classificadas como infecção incisional superficial; $52,9 \%$, infecção incisional profunda e 23,5\%, infecção de órgão/espaço; $41,2 \%$ dos casos de infeç̧ão desenvolveram-se no período de 6 a 10 dias após a cirurgia e foram isolados diferentes microrganismos nas culturas solicitadas. Evidenciou-se que a freqüência de ISC em cirurgia potencialmente contaminada está de acordo com o encontrado na literatura. Entretanto, em relação à topografia, a maioria dos casos detectados foi classificada como infecção incisional profunda e a literatura retrata que dois terços das infecções são confinados à incisão.
\end{abstract}

Palavras-chave: Infecção da ferida operatória. Gastrectomia. Enfermagem Perioperatória.

\section{Abstract}

This research aimed to identify the frequency of surgical site infection (SSI) in patients submitted to potentially contaminated, elective gastrectomy, at the Gastric Surgery specialty of a school hospital, in the period from 1998 to 2002. A retrospective study was carried out by means of an information survey in medical records. Surgical site infection was detected in 17 patients $(9.4 \%)$ out of 181 analyzed cases, $23.5 \%$ of which were classified as superficial incisional infection; $52.9 \%$ as profound incisional infection and $23.5 \%$ as organ/space infection; $41.2 \%$ of the infection cases developed between 6 and 10 days after the surgery and different microorganisms were isolated in the requested cultures. It was evidenced that the frequency of SSI in potentially contaminated surgeries is according to literature. However, what topography is concerned, most of the detected cases were classified as profound incisional infection, while literature reports that two thirds of SSI are confined to the incision.

Keywords:

Surgical Wound Infection. Gastrectomy.

Perioperative Nursing.

\section{Resumen}

La finalidad de esta investigación fue identificar la frecuencia de infección de herida operatoria, en paciente sometido a cirugía electiva de gastrectomía, potencialmente contaminada, en la especialidad de Gastrocirugía, durante el período de 1998 y 2002, en un hospital escuela de Ribeirão Preto São Paulo - Brasil. Fue efectuado un estudio retrospectivo, por medio de la recopilación de informaciones contenidas en los archivos médicos. En 181 casos investigados fue detectada la ocurrencia de infección de incisión quirúrgica en 17 pacientes $(9,4 \%), 23,5 \%$ de las cuales fueron clasificadas como infección incisional superficial; 52,9\% como infección incisional profunda y $23,5 \%$ como infección de órgano/ espacio; $41,2 \%$ de los casos de infección se desarrollaron en el período de 6 a 10 días después de la cirugía y fueron aislados diferentes microorganismos en las culturas solicitadas. Se evidenció que la frecuencia de infecciones en cirugía potencialmente contaminada está de acuerdo con lo que es preconizado por la literatura. Sin embargo, respecto a la topografía, la mayoría de los casos detectados fue clasificada como infección incisional profunda y la literatura retrata que dos tercios de las infecciones son confinados a la incisión.

Palabras clave:

Infección da Herida Operatoria. Gastrectomía.

Enfermería Perioperatoria. 


\section{INTRODUÇÃO}

A infecção hospitalar ( $(H)$ constitui-se em grave problema de saúde pública no Brasil e no mundo, sendo uma das principais causas de morbidade e letalidade de pessoas submetidas a procedimentos clínicos de assistência'.

Entre as infecções hospitalares, salientamos a infecção do sítio cirúrgico (ISC). Segundo o Centers for Disease Control and Prevention (CDC), o diagnóstico desse tipo de infecção é feito observando-se os seguintes critérios: 0 surgimento da infecção deve acontecer até 30 dias após o procedimento cirúrgico, ou, em casos de implante de prótese, em até 1 ano. Em relação à topografia, ela é classificada em: infecção incisional superficial, quando envolve apenas pele e tecido subcutâneo do local da incisão cirúrgica; infecção incisional profunda, quando acomete obrigatoriamente tecidos moles profundos (fáscia e camadas musculares) e infecção orgão/ espaço específica, quando a infecção puder ser relacionada à operação ou envolver qualquer parte da anatomia, aberta ou manipulada durante a cirurgia, mas não necessariamente a incisão cirúrgica².

De acordo com dados do Study on the Efficacy of Nosocomial Infection Control (SENIC), publicado em 1980, em termos de incidência, a ISC é superada apenas pela infecção do trato urinário ${ }^{3}$.

Entre os pacientes cirúrgicos, a ISC é a infecção hospitalar mais comum, sendo que dois terços delas são confinadas à incisão, e um terço envolve órgãos e espaços abordados durante o procedimento. Quando os pacientes cirúrgicos com ISC morrem, 77\% dessas mortes estão relacionadas à infecção, e a maioria (93\%) envolve órgãos e espaços manipulados na cirurgia².

A instalação ou não da ISC depende de diferentes aspectos, mas principalmente do inóculo do microrganismo e sua virulência, bem como da capacidade de defesa do hospedeiro. Assim, os fatores que influenciam a incidência da ISC podem ser divididos em três categorias, ou seja, relacionados ao microrganismo, paciente e ao procedimento cirúrgico ${ }^{4}$.

A contaminação microbiana do sítio cirúrgico é um aspecto precursor da infecção. No entanto, o local contaminado deve conter um inóculo maior que 10 microrganismos por grama de tecido ${ }^{5}$. Ressaltamos que a maioria das ISC é causada pela microbiota endógena do paciente, oriunda freqüentemente da pele, mucosas e vísceras².

A literatura aponta diferentes fatores relacionados ao paciente, tais como: a presença de doenças crônicas, como o diabetes mellitus e a obesidade, o tabagismo e 0 etilismo. Entre os fatores relacionados ao procedimento cirúrgico, ressalta-se o período de internação, a duração da cirurgia, a técnica cirúrgica e a utilização de transfusão sanguínea. Estes fatores, mencionados em estudos recentes, foram considerados como contribuidores para 0 aumento da incidência de ISC ${ }^{5-13}$.

Em 1964, o National Research Council ad hoc Committee on Trauma, elaborou uma classificação de cirurgias por potencial de contaminação, que tinha como finalidade principal estabelecer riscos aceitáveis de ISC'.

Essa classificação tem sofrido alterações, mas os conceitos principais têm se mantido. Assim, as cirurgias podem ser classificadas em: (1) limpa (cirurgias eletivas com cicatrização de primeira intenção realizadas na ausência de processo inflamatório e de falhas nas técnicas assépticas, sem drenos, em que não ocorram penetrações nos tratos gastrintestinal, respiratório, geniturinário ou orofaringe); (2) potencialmente contaminada (cirurgias realizadas na ausência de processo inflamatório e com falhas pequenas de técnicas. Ocorre penetração nos tratos gastrintestinal, respiratório ou geniturinário sob condições controladas; ressaltamos que a gastrectomia inclui-se nesta categoria); (3) contaminada (cirurgias realizadas em tecidos traumatizados há menos de quatro horas; aquelas em que tenham ocorrido falhas grosseiras da técnica asséptica e presença de inflamação não purulenta aguda na incisão, contaminação do trato gastrintestinal); e (4)suja ou infectada (qualquer cirurgia realizada na presença de secreção purulenta, ferida traumática com tecido desvitalizado, presença de corpo estranho ou contaminação fecal, trauma penetrante há mais de quatro horas, perfuração de víscera). Portanto, as taxas de ISC devem ser proporcionais ao potencial de contaminação, ou seja, taxas menores nas cirurgias limpas e maiores nas sujas ou infectadas'.

Procurando contribuir e somar esforços para a construção de conhecimento que possibilite fundamentar - trabalho dos profissionais de saúde na implementação de ações eficazes na prevenção e 0 controle da $\mathrm{IH}$, propomos o presente estudo com 0 objetivo de identificar a freqüência da ISC em gastrectomia (cirurgia potencialmente contaminada) em um hospital escola, no período de 1998 a 2002.

\section{PROCEDIMENTO METODOLÓGICO}

A presente investigação é um estudo retrospectivo, com abordagem quantitativa e delineamento de pesquisa não-experimental.

0 local selecionado para o estudo foi um hospital regional, de nível terciário, situado no interior do Estado de São Paulo e destinado ao ensino, pesquisa e assistência. Ele é composto por ambulatórios, unidades de internação, laboratórios e serviços especializados, administração, unidades de apoio, entre outros setores. 
Conta ainda com 847 leitos, atendendo à demanda proveniente de 21 cidades da região, sendo responsável por $20 \%$ dessas internações. Segundo informações do Grupo de Avaliação de Desempenho, no ano de 2003, o Hospital foi responsável por 29.367 cirurgias e 32.807 internações, tendo a especialidade de gastrocirurgia realizado 572 procedimentos cirúrgicos nesse mesmo ano ${ }^{14}$.

Para o alcance do objetivo proposto, elaboramos um instrumento, que foi submetido à análise, para validação aparente e de conteúdo, de três juízes (um docente de universidade e dois enfermeiros de hospital público) com experiência na temática investigada. 0 instrumento elaborado continha dados relacionados à identificação do paciente como, por exemplo: idade, sexo, entre outros. Para classificarmos a topografia dos casos de ISC descritos nos prontuários médicos, utilizamos os critérios preconizados pelo Centers for Disease Control and Prevention em 1999².

Após a validação aparente e de conteúdo, realizamos o teste piloto utilizando 10 prontuários médicos que não estavam incluídos no período proposto para a coleta de dados, para verificarmos a adequação do instrumento frente à mensuração do pretendido.

Solicitamos 253 prontuários médicos, que foram separados a partir de listagens previamente agendadas no Serviço de Arquivo Médico (SAME), onde realizamos a coleta de dados.

Não pudemos realizar a revisão de 12 prontuários, pois, de acordo com informações do arquivo médico, eles estariam sendo utilizados no atendimento aos pacientes, ou em posse de algum médico do hospital. Excluímos os prontuários cujas cirurgias foram realizadas em caráter de urgência e aqueles cuja proposta inicial diferiam da realizada, totalizando 60 prontuários médicos.

Assim, dos 253 prontuários médicos solicitados ao SAME, 181 estavam de acordo com os critérios de inclusão no estudo, ou seja, os pacientes foram submetidos a gastrectomias eletivas no período compreendido entre janeiro de 1998 a dezembro de 2002, cujos prontuários médicos estavam disponíveis no SAME do Hospital escolhido para o estudo. Após a seleção dos prontuários, realizamos a coleta de dados nos meses de maio, junho e julho de 2003.

Destacamos que, na presente investigação, analisamos apenas as cirurgias eletivas, pois entendemos que as cirurgias consideradas emergenciais possuem características que propiciam maior risco de infecção, tais como: não há geralmente tempo de compensar doenças de base, avaliar focos infecciosos à distância ou estado nutricional do paciente, o profissional pode cometer descuidos na técnica e na preparação do paciente, expondo-o a maior probabilidade de desenvolver infecção, pois quando o risco de morte é iminente, a infecção passa a ser um tributo que a equipe de saúde paga para manter a vida do paciente 4 .

Este projeto de pesquisa foi submetido à apreciação do Comitê de Ética em Pesquisa do Hospital selecionado, sendo aprovado de acordo com a Resolução 196/96, do Conselho Nacional de Saúde, que regulamenta a pesquisa em seres humanos ${ }^{15}$. Foi dispensada a aplicação do termo de consentimento livre e esclarecido, já que os dados foram coletados nos prontuários médicos de cada paciente.

\section{RESULTADOS}

\section{Caracterização dos sujeitos investigados}

Dos sujeitos participantes do estudo (181 pacientes), 65 indivíduos eram do sexo feminino e 116 do masculino, sendo 140 da raça branca, 23 mulatos, 15 negros e 3 da raça amarela.

Em relação à idade, 26,5\% (48) dos sujeitos investigados estavam na faixa etária entre 61 e 70 anos; $22,6 \%$ (41) na faixa etária entre 51 e 60 anos; 18,8\% (34) na faixa etária entre 71 e 80 anos; $13,8 \%$ (25) na faixa etária entre 41 e 50 anos; 10,5\% (19) na faixa etária entre 31 e 40 anos; 4,4\% (8) na faixa etária entre 20 e 30 anos e $3,3 \%$ (6) com 81 anos ou mais.

Do total da amostra, $149(82,5 \%)$ sujeitos apresentaram diagnóstico médico oncológico.

Observamos que, entre os sujeitos investigados, 164 $(90,6 \%)$ não desenvolveram ISC e 17 (9,4\%) deles desenvolveram-na, sendo 15 deles do sexo masculino e apenas 2 do feminino. A raça branca predominou, apresentando 16 casos de infecção, 12 dos quais com diagnóstico médico oncológico. Ressaltamos que, quanto à idade, $7(41,1 \%)$ indivíduos estavam na faixa etária entre 61 e 70 anos; 3 $(17,6)$ na faixa etária entre 31 e 40 anos; $3(17,6)$ na faixa etária entre 41 e 50 anos; $3(17,6)$ na faixa etária entre 51 e 60 anos e $1(5,9)$ na faixa etária entre 71 e 80 anos.

\section{Frequiência de ISC}

Por meio da revisão dos prontuários médicos constatamos $17(9,4 \%)$ casos de infecção do sítio cirúrgico, sendo que $4(23,5 \%)$ eram infecção incisional superficial; 9 (52,9\%), decorreram de infecção incisional profunda e 4 (23,5\%), de infecção de órgão/espaço.

Dos 17 casos de ISC constatados, $14(82,3 \%)$ foram diagnosticados durante a internação e $3(17,6 \%)$, no período de retorno ambulatorial.

Conforme podemos observar na Tabela 1, $7(41,2 \%)$ dos casos de infecção detectados desenvolveram-se no período de 6 a 10 dias após a cirurgia; 5 casos $(29,4 \%)$ entre 1 a 5 dias; 3 casos (17,6\%) entre 16 e 30 dias; e 2 casos $(11,8 \%)$ entre 11 e 15 dias. 
Tabela 1:

Distribuição dos pacientes submetidos a gastrectomias em um hospital escola segundo o período entre a cirurgia e o diagnóstico de ISC (dias). Ribeirão Preto, 2003.

\begin{tabular}{c|c|c}
\hline $\begin{array}{c}\text { Período entre a cirurgia e o } \\
\text { diagnóstico de ISC (dias) }\end{array}$ & N & $\%$ \\
\hline 1 a 5 & 05 & 29,4 \\
6 a 10 & 07 & 41,2 \\
11 a 15 & 02 & 11,8 \\
16 a 30 & 03 & 17,6 \\
\hline TOTAL & 17 & 100 \\
\hline
\end{tabular}

\section{Microrganismos isolados}

De acordo com a Tabela 2, nos 17 casos de ISC, em 8 deles (47\%) não houve solicitação de cultura do microrganismo, enquanto nos restantes (53\%) foram identificados os seguintes microrganismos: Staphylococcus aureus, Staphylococcus epidermidis, Enterobacter faecis, Enterobacter cloacae, Escherichiae coli, Staphylococcus bluolerico, Streptococcus pneumoniae, Streptococcus agalactaeae, Bacteroides fragilis, Pseudomonas aeruginosa. Em 5 dos casos de ISC diagnosticados, as culturas apontaram o crescimento de mais de um microrganismo.

\section{Tabela 2:}

Distribuição dos microrganismos isolados nas culturas dos pacientes submetidos a gastrectomias em um hospital escola. Ribeirão Preto, 2003.

\begin{tabular}{|c|c|c|}
\hline $\begin{array}{l}\text { Microorganismos isolados } \\
\text { por cultura }\end{array}$ & $\mathrm{N}$ & $\%$ \\
\hline $\begin{array}{l}\text { Escherichiae coli, Enterobacter } \\
\text { cloacae }\end{array}$ & 01 & 5,9 \\
\hline Staphylococcus aureus & 01 & 5,9 \\
\hline $\begin{array}{l}\text { Staphylococcus aureus, } \\
\text { Enterobacter cloacae }\end{array}$ & 01 & 5,9 \\
\hline $\begin{array}{l}\text { Staphylococcus bluolerico, } \\
\text { Streptococcus pneumoniae, } \\
\text { Bacteroides fragilis }\end{array}$ & 01 & 5,9 \\
\hline $\begin{array}{l}\text { Staphylococcus epidermidis, } \\
\text { Enterobacter faecis }\end{array}$ & 01 & 5,9 \\
\hline $\begin{array}{l}\text { Streptococcus agalactaeae, } \\
\text { Escherichiae coli }\end{array}$ & 01 & 5,9 \\
\hline Pseudomonas aeruginosa & 02 & 11,8 \\
\hline Microrganismo indeterminado & 01 & 5,9 \\
\hline Ausência de cultura & 08 & 47,0 \\
\hline TOTAL & 17 & 100 \\
\hline
\end{tabular}

\section{DISCUSSÃO}

Segundo estudo do CDC, a taxa média de ISC esperada para cirurgias potencialmente contaminadas é de 3 a $11 \%$, sendo que a taxa de infecção para feridas consideradas limpas é de 1 a 5\%, o que aponta a contaminação endógena como fator principal no desenvolvimento de infecção. As taxas elevadas de infecção em cirurgias limpas podem indicar quebra da técnica asséptica, servindo como índice de comparação entre hospitais e equipes, guardadas as devidas ressalvas ${ }^{16}$.

Neste estudo, do total de 181 pacientes investigados, 17 (9,4\%) deles desenvolveram ISC, cujo índice de infecção encontra-se dentro da taxa média divulgada pelo CDC para cirurgias potencialmente contaminadas.

Em um estudo nacional foram analisados 134 sujeitos submetidos a diferentes procedimentos cirúrgicos na especialidade de gastrocirurgia, no período de um ano. Os autores constataram 13,4\% (18 casos) de freqüência de ISC, sendo que $50 \%$ (9 casos) consideraram infecção incisional superficial, 44,4\% (8 casos) infecção incisional profunda e 5,5\% (1 caso) infecção órgão/espaço ${ }^{17}$.

A esse respeito, avaliando cirurgias consideradas limpas, em outra pesquisa nacional, estudo tipo coorte, os autores analisaram 305 pacientes submetidos a artroplastia de quadril, e concluíram que em 26 procedimentos ocorreu ISC, sendo que $88,5 \%$ delas foram consideradas superficiais (incidência de 7,5\%) e 11,5\%, foram classificadas como profundas (incidência de $0,98 \%)^{10}$.

Outro estudo analisou 4718 pacientes submetidos a cirurgias abdominais não cólon-retais. Os autores verificaram que em relação a cirurgias consideradas limpas, o índice de infecção foi de 0,9\%; quanto às cirurgias potencialmente contaminadas ele foi de $1,6 \%$; em cirurgias consideradas contaminadas foi de $2,7 \%$ e foi de $3,4 \%$ para cirurgias infectadas ${ }^{11}$.

Em relação a cirurgias contaminadas, em um estudo que analisou 2809 pacientes submetidos a cirurgias eletivas de ressecção cólon-retal, verificou-se que a incidência de ISC em geral foi de 4,7\% (134 casos de infecção diagnosticados) ${ }^{18}$.

Quanto à vigilância pós-alta, a literatura ressalta sua importância, pois os casos de infecção seriam subestimados se ela não existisse, como podemos verificar em estudos, nos quais os autores identificaram que $32,5 \%$ dos casos de infecção foram diagnosticados no ambulatório durante o retorno do paciente ${ }^{19}$. Em outra pesquisa, os estudiosos verificaram que $62,9 \%$ das infecções do sítio cirúrgico foram notificadas no ambulatório de egressos, sendo que a maioria das infecç̃es (89,7\%) foi notificada até 21 dias após a cirurgia ${ }^{20}$.

Nossos dados corroboram esses estudos, uma vez que houve predomínio do diagnóstico de ISC $(41,2 \%)$ no período de 6 a 10 dias após a cirurgia. 
Estudiosos demonstraram que, dos 26 casos de infecção diagnosticados, $20(76,9 \%)$ ocorreram durante 0 período de internação e $6(23,1 \%)$ deles foram detectados no período pós-alta, lembrando que todas essas infecç̃es foram diagnosticadas em um período de 30 dias $^{10}$.

Atualmente, em virtude da alta cada vez mais precoce $e$ de procedimentos ambulatoriais, faz-se necessário a adoção de medidas de vigilância que extrapolem os portões dos hospitais, lembrando que a subnotificação não apenas traduz taxas irreais de infecção de um determinado serviço, mas também impede a implementação de medidas eficazes para 0 controle e prevenção de suas complicações ${ }^{20}$.

No presente estudo não foi possível determinar o microrganismo causador do processo infeccioso em todos os casos diagnosticados. No entanto, os microrganismos encontrados são os mais relatados na literatura. Outra pesquisa também constatou que, em 26 casos de infecção detectados, apenas em 15 solicitaram-se culturas, portanto, em 11 casos não foi possível analisar o microrganismo causador da ISC ${ }^{10}$.

Como principais causadores de infecção hospitalar, nos Estados Unidos da América (EUA), em primeiro lugar está E. coli, seguido pelo $\mathrm{S}$. aureus e, em terceiro, $\mathrm{S}$. epidermidis ${ }^{21}$.

Quanto à transmissão dessas bactérias, as narinas dos profissionais de saúde estão predispostas à colonização pelo $\mathrm{S}$. aureus. Eles comumente, podem transferilo aos pacientes através de suas mãos transitoriamente colonizadas. Em uma pesquisa, o autor investigou a colonização bacteriana por $\mathrm{S}$. aureus em alunos de um curso de auxiliar de enfermagem, em diferentes momentos de sua formação profissional, e concluiu que a cavidade nasal foi a área anatômica mais colonizada, seguida da mão direita e mão esquerda ${ }^{22}$.

Outro aspecto importante a ser ressaltado foi a resistência crescente desse microrganismo aos antimicrobianos utilizados para seu tratamento, com cepas meticilino-resistentes (MRSA), que dificultam seu tratamento. Portanto, devemos evitar a disseminação intra-hospitalar, por meio da implementação de medidas preventivas como a lavagem das mãos com soluções preconizadas, precauções de contato para pacientes com lesões cutâneas, infectados ou colonizados ${ }^{21}$.

Dessa maneira, a utilização da antibioticoterapia pelos profissionais da saúde, desde sua prescrição até sua administração, contribui de maneira significativa para 0 aumento da resistência bacteriana ${ }^{23}$.

Verificamos que os microrganismos identificados no presente estudo são também freqüentemente isolados em outras instituições e em outros tipos de procedimentos, como no estudo que os autores por meio de um programa de vigilância para avaliação da incidência e natureza das infecções, analisaram 40195 cirurgias até um mês após o procedimento durante 10 anos. Eles verificaram a ocorrência de 1032 ISC. Em relação às culturas obtidas em $95 \%$ das incisões infectadas, houve crescimento de microrganismo em $97,5 \%$ delas. 0 microrganismo mais isolado em cirurgias limpas foi 0 Staphylococcus aureus, seguido pelos enterococcus e Pseudomonas. Em cirurgias potencialmente contaminadas foram encontrados mais freqüentemente enterococcus e em cirurgias consideradas contaminadas o crescimento de Pseudomonas foi mais freqüente, seguido por enterococcus e $S$. aureus ${ }^{24}$.

Estudiosos procuraram analisar retrospectivamente 1980 pacientes submetidos a cirurgias cardíacas diagnosticando 37 infecções profundas $(1,9 \%)$ e 46 infecções superficiais $(2,3 \%)$. Os autores verificaram também que menos da metade das infecções superficiais tiveram culturas coletadas. Quanto aos microrganismos encontrados, o $\mathrm{S}$. aureus foi isolado em $54 \%$ das infecções profundas e em $41 \%$ dos pacientes com infecção incisional superficial. Ressaltaram também que foram isolados bacilos gram-negativos em aproximadamente um quarto dos pacientes ${ }^{25}$.

Assim, em relação à etiologia da ISC, autores fundamentados na literatura descrevem que qualquer agente infeccioso pode ser responsável por este tipo de infecção. No geral, ela é causada por agentes presentes na pele ou nas mucosas do próprio paciente. Nas cirurgias limpas, os cocos gram-positivos são mais isolados enquanto nos procedimentos potencialmente contaminados e contaminados são mais freqüentes as bactérias gram-negativas aeróbicas e anaeróbicas ${ }^{4}$.

Salientamos que apesar de grande parcela da ISC ser causada pela microbiota endógena, portanto proveniente do próprio paciente, é importante ressaltar a contribuição da equipe e do ambiente inanimado, que também podem ser carreadores de microrganismos, destacando a importância da implementação de medidas eficazes para a prevenção e o controle desse tipo de infecção. Além desses aspectos, ressaltamos os fatores predisponentes relacionados ao paciente e ao procedimento cirúrgico comprovados na literatura que aumentam o risco para 0 desenvolvimento da ISC ficando claro a característica multicausal dessa complicação.

\section{CONCLUSÃO}

A freqüência evidenciada de ISC em gastrectomia no hospital selecionado para o presente estudo está dentro do estimado pelo CDC. A porcentagem diagnosticada foi de $9,4 \%$ e a taxa média de ISC esperada para cirurgias potencialmente contaminadas é 3 a $11 \%{ }^{16}$. 
A vigilância pós-alta não detecta apenas os casos de infecção que se desenvolvem fora do ambiente hospitalar. Permite também, a avaliação da eficácia das medidas implementadas no serviço para a prevenção e o controle de $\mathrm{H}$. Assim, entendemos a importância da implantação de programas de vigilância pós-alta, os quais proporcionam taxas confiáveis de $\mathrm{HH}$, além da melhoria da assistência prestada ao paciente.

Em relação às culturas, a maioria dos gêneros evidenciados neste estudo constitui-se em microrganismos próprios da microbiota humana que, em virtude da crescente resistência microbiana ou de serem oportunistas, se aproveitam do estado imunodeprimido do hospedeiro, tornando-se capazes de causar infecção.

\section{Referências}

1. Lacerda RA. Infecção hospitalar e sua relação com a evolução das práticas de assistência à saúde. In: Lacerda RA, organizadora. Controle de infecção em centro cirúrgico: fatos, mitos e controvérsias. São Paulo (SP): Atheneu; 2003.

2. Mangram AJ, Horan TC, Pearson ML, Silver LC, Jarvis WR. Guideline for prevention of surgical site infection. AllC 1999 Apr; 27 (2): 97-132.

3. Grinbaum RS. Infecção do sítio cirúrgico e antibioticoprofilaxia em cirurgia. In: Rodrigues EAC, organizador. Infecções hospitalares, prevenção e controle. São Paulo (SP): Sarvier; 1997.

4. Rabhae GN, Ribeiro Filho N, Fernandes AT. Infecção do sítio cirúrgico. In: Fernandes AT, Fernandes MOV, Ribeiro Filho N. Infecção hospitalar e suas interfaces na área da saúde. São Paulo (SP): Atheneu; 2000.

5. Estrada CA, Young JA, Nifong LW, Chitwood RJ. Outcomes and perioperative hyperglycemia in patients with or without diabetes mellitus undergoing coronary artery bypass grafting. Ann Thorac Surg 2003 May; 75 (5): 1392-99.

6. Kim J, Hammar N, Jakobsson K, Luepker RV, Mcgovern PG, Torbjorn I. Obesity and the risk of early and late mortality after coronary artery bypass graft surgery. Am Heart J 2003 Sep; 146 (3): 555-60.

7. Delgado-Rodríguez M et al. A prospective study of tobacco smoking as a predictor of complications in general surgery. Infect Control Hosp Epidemiol 2003 Jan; 24 (1): 37-43.

8. Delgado-Rodríguez M et al. Alcohol consumption and the risk of nosocomial infection in general surgery. Br J Surg 2003 Oct; 90 (10): 1287-93.

9. Sorensen LT, Horby J, Friis E, Pilsgaard B, Jorgensen T. Smoking as a risk factor for wound healing and infection in breast cancer surgery. Eur J Surg Oncol 2002 Dec; 28 (8): 815-20.
Os microrganismos encontrados no estudo são freqüentemente isolados em infecções de diversos sítios e procedimentos. Assim, faz-se necessário o controle e atuação consciente dos profissionais de saúde no que tange à microbiota responsável pelas infecções dentro do hospital. Cabe a Comissão de Controle de Infecção Hospitalar $(\mathrm{CClH})$, pautada na realidade específica daquela instituição, escolher o melhor esquema de antibioticoterapia.

Ressaltamos ainda que na presente pesquisa, os dados foram obtidos por meio dos registros efetuados nos prontuários médicos, ou seja, dependentes da qualidade das informaç̧ões registradas pelos profissionais de saúde envolvidos no atendimento ao paciente cirúrgico.

10. Ercole FF, Chianca TCM. Infecção do sítio cirúrgico em pacientes submetidos a artroplastias de quadril. Rev Latino-am Enfermagem $2002 \mathrm{mar} / \mathrm{abr} ; 10$ (2): 157-65.

11. Pessaux P, Msika S, Atalla D, Hay JM, Flamant Y. Risk factors for postoperative infectious complications in noncolorectal abdominal surgery: a multivariate analysis based on a prospective multicenter study of 4718 patients. Arch Surg 2003 Mar; 138 (3): 314-24.

12. Wurtz $R$ et al. Do new surgeons have higher surgical-site infection rates? Infect Control Hosp Epidemiol 2001 Jun; 22 (6): 375-77.

13. Morris CD, Sepkowitz K, Fonshell C, Margetson N, Eagan J, Miransky J et al. Prospective identification of risk factors for wound infection after lower extremity oncologic surgery. Ann Surg Oncol 2003 Aug; 10 (7): 778-82.

14. Hospital das Clínicas(SP). Grupo de Avaliação de Desempenho. Relatório de avaliação de desempenho. Ribeirão Preto (SP); 2003.

15. Resolução ${ }^{0} 196$ de 10 de outubro de 1996. Diretrizes e normas regulamentadoras de pesquisas envolvendo seres humanos. [on line] [citado 22 mar 2004]. Disponível em http:// www.conselho.saude.gov.br/docs/resoluçoes/resol96.doc.

16. Garner IS. CDC guidelines for the prevention and control of nosocomial infections: guideline for surgical wound infections. Am J Infect Control 1986; 14: (71-80).

17. Poveda VB, Galvão CM, Hayashida M. Análise dos fatores de risco relacionados à incidência de infecção do sítio cirúrgico em gastrocirurgias. Rev Esc Enferm USP 2003 mar; 37 (1): 81-9.

18. Tang R, Chen HH, Wang YL, Changchien CR, Chen JS, Hsu KC et al. Risk factors for SSI after elective resection of the colon and rectum: a single-center prospective study of 2809 consecutive patients. Ann Surg 2001 Aug; 234 (2): 181-89. 
19. Lemos FN, Oliveira AV, Sousa MG. Infecção de sítio cirúrgico: estudo prospectivo de 2149 pacientes operados. Rev Col Bras Cirurgiões 1999 mar/abr; 26 (2): 109-13.

20. Oliveira AC, Martins MA, Martinho GH, Clemente WT, Lacerda RA. Estudo comparativo do diagnóstico da infecção do sítio cirúrgico durante e após a internação. Rev Saúde Pública 2002 dez; 36 (6): 717-22.

21.Fernandes AT, Ribeiro Filho N, Mazzano RS, Santana LB, Cerbara EFV, Cassaro Júnior E. Bactérias aeróbias. In: Fernandes AT, Fernandes MOV, Ribeiro Filho N. Infecção hospitalar e suas interfaces na área da saúde. São Paulo (SP): Atheneu; 2000.

22. Oliveira Santos BM. 0 portador são de Staphylococcus aureus: um estudo longitudinal em alunos de um curso de auxiliar de enfermagem [tese de livre docência]. Ribeirão Preto (SP): Escola de Enfermagem /USP; 1996.

23. Albuquerque VS, Campos ECB, Souza RM. Antibioticoterapia: "a queda das pastilhas". Esc Anna Nery Rev Enferm 1997 set; 1(1): 131-40.

24. Olson MM, Lee JT. Continuous, ten years wound infection surveillance: results, advantages and unanswered questions. Arch Surg 1990; 125: 794-803.

25. Olsen MA, Lock-Buckley P, Hopkins D, Polish LB, Sundt TM, Fraser VJ. The risk factors for deep and superficial chest surgical site infection alter coronary artery bypass graft surgery are different. J Thorac Cardiovasc Surg 2002 Jul; 124 (1): 136-45.

\section{Notas}

Este trabalho é parte da dissertação de mestrado de Poveda VB. Análise dos fatores predisponentes à infecção do sítio cirúrgico em gastrectomia [dissertação de mestrado]. Ribeirão Preto (SP): Escola de Enfermagem de Ribeirão Preto/USP; 2004.

\section{Sobre as Autoras}

\section{Vanessa de Brito Poveda}

Enfermeira. Doutoranda do Programa de Pós-Graduação Enfermagem Fundamental do Departamento de Enfermagem Geral e Especializada da Escola de Enfermagem de Ribeirão Preto da Universidade de São Paulo (DEGE-EERP/USP).

\section{Cristina Maria Galvão}

Enfermeira. Doutora em Enfermagem e Professora Associada do DEGE-EERP/USP. E-mail: crisgalv@eerp.usp.br 